\title{
Fingerprinting techniques investigation to detect petroleum hydrocarbon origin in coastal sediments of Persian Gulf
}

\begin{abstract}
Persian Gulf coasts are subjected to different sources of petroleum pollution. This paper enhanced fingerprinting method of applying biomarkers polycyclic aromatic hydrocarbons (PAHs) and nalkanes in identifying the distribution of oil spills and petroleum products. A total of 18 samples were collected from the northern coasts of Persian Gulf. PAH concentrations in the coastal sediments ranged from 184.49 to $2771.88 \mathrm{ng}$ g-1. Distribution of three-ring PAHs was much higher than the others. PAHs concentration can be categorized as low to moderate comparing the global scale. Applying principal component analysis and data clustering revealed two major groups. The first group, including alkylated phenanthrenes, belongs to the low molecular weight PAHs with two and three rings, which are abundant in petrogenic sources mostly caused by petroleum spills. The second group consists of four- and five-ring PAHs. This group is frequently detected in pyrogenic source, such as combustion of coal, wood, vehicle fuel, and waste tire.
\end{abstract}

Keyword: N-alkanes; Nickel; Persian Gulf; Polycyclic aromatic hydrocarbons; Vanadium 